\title{
Carcinoma Verrucoso Oral: Reporte de un Caso Clínico y Revisión de 20 Casos del Instituto de Referencia en Patología Oral (IREPO), Chile
}

\author{
Oral Verrucous Carcinoma: A Case Report and Review of 20 Cases of the Oral \\ Pathology Referral Institute (IREPO), Chile
}

\author{
Adorno Farias $\mathrm{D}^{1}$, Maturana Ramírez $\mathrm{A}^{2}$, Farías Vergara $\mathrm{M}^{2}$, Franco Martínez $\mathrm{ME}^{3}$, Iriarte Hernández $\mathrm{M}^{4}$, \\ Sáez Salgado $\mathrm{R}^{5}$, Cortés Araya $\mathrm{J}^{6}$, Ortega Pinto $\mathrm{AV}^{3}$
}

\section{RESUMEN}

El carcinoma verrucoso (CV) es una variante rara del carcinoma de células escamosas con características morfológicas y comportamiento específico. El presente estudio relata el caso de una paciente de género femenino, de 68 años de edad, que presenta un carcinoma verrucoso en lengua, indoloro y con 8 meses de evolución. Además, se realizó una breve revisión de casos clínicos del Instituto de Referencia en Patología Oral (IREPO) de la Facultad de Odontología de la Universidad de Chile, diagnosticados entre enero de 1984 y octubre de 2010, encontrándose 20 casos, con un promedio de edad de 70 años, localizados con mayor frecuencia en encía inferior y lengua.

Rev. Clin. Periodoncia Implantol. Rehabil. Oral Vol. 3(3); 132-135, 2010.

Palabras clave:Carcinoma verrucoso, caso clínico.

\section{ABSTRACT}

Verrucous carcinoma (VC), a rare variant of squamous cell carcinoma is an established entity with distinctive morphology and specific clinical behavior. The present study describe a case report of a 68-year-old women who presented a tongue verrucous carcinoma, asymptomatic, that had about 8 months of evolution. A brief review of VC cases diagnosed in Oral Pathology Referral Institute (IREPO), Faculty of Odontology, University of Chile, between 1984 and 2010. It was found 20 cases of verrucous carcinoma with a median age of 70 -years-old, the most common places were lower gingiva and tongue.

Rev. Clin. Periodoncia Implantol. Rehabil. Oral Vol. 3(3); 132-135, 2010.

Key words: Verrucous carcinoma, case report.

\section{INTRODUCCIÓN}

El carcinoma verrucoso (CV) es una variante del carcinoma de células escamosas (CCE) bien diferenciado, que se caracteriza por ser exofítico con múltiples prominencias y profundas hendiduras ${ }^{(1)}$. La cavidad oral es el sitio más prevalente de esta patología en cabeza y cuello, representando el $75 \%$ de los casos $^{(2)}$, seguido de la laringe con un $15 \%-35 \%$ de todos los $C V^{(1)}$. Lesiones similares han sido descritas en sitios como piel, región cérvico-uterina, vejiga, región ano-rectal, región genital y esófago ${ }^{(2,3)}$.

En cavidad bucal se encuentra principalmente en encía, mucosa alveolar y yugal(2). Presenta mayor predilección por el sexo masculino, en la sexta y séptima década de vida ${ }^{(1,2,3)}$.

Considerado como un carcinoma con capacidad limitada de invadir y dar metástasis, en cavidad oral el CV representa solamente del $2 \%$ al $12 \%$ de todas las neoplasias epiteliales. Este tumor es de crecimiento lento, es agresivo localmente, pero bien delimitado. Linfonodos cervicales están palpables en muchos casos y metástasis son muy poco frecuentes ${ }^{(1,2)}$

De etiología desconocida, el CV ha sido asociado al consumo de tabaco crónico en todas sus variedades y al virus papiloma humano, genotipos 16, $18 \mathrm{y}$, raramente, 6 y 11, que han sido identificados en algunos $\mathrm{CV}^{(1)}$.

Histológicamente el CV se caracteriza por la presencia de un epitelio extremadamente engrosado, con papilas bulbosas que se invaginan hacia el estroma y superficie con marcada hiperparaqueratinización. La lesión es diferenciada, habitualmente no presenta atipias, pudiendo encontrarse displasia leve. La membrana basal se encuentra íntegra y se observan cambios inflamatorios crónicos en el conectivo subyacente ${ }^{(1,2)}$.

El diagnóstico diferencial de CV incluye: CCE exofítico, CV híbrido, CCE papilar, papiloma de células escamosas queratinizantes y verruga vulgar. El CV tiene un excelente pronóstico, con una tasa de supervivencia a los cinco años, en laringe, de 85\%-95\%. El tratamiento es la excisión quirúrgica con láser o cirugía convencional. En casos de pacientes que no puedan ser sometidos a cirugía, se acepta la radioterapia como una alternativa de tratamiento(1).

El objetivo de este estudio fue reportar los aspectos clínicos e histopatológicos de un caso de carcinoma verrucoso oral en lengua y describir los casos diagnosticados por el Instituto de Referencia en Patología Oral (IREPO) entre el periodo de enero de 1984 hasta octubre de 2010.

\section{REPORTE DEL CASO}

Se presenta una paciente de género femenino de 68 años de edad que consulta al Servicio de Diagnóstico de la Facultad de

\footnotetext{
1. Profesora Asistente. Departamento de Patología Oral e IREPO. Facultad de Odontología, Universidad de Chile. Chile.

2. Instructor. Departamento de Patología Oral. Facultad de Odontología, Universidad de Chile. Chile.

3. Profesora Asistente. Departamento de Patología Oral. Facultad de Odontología, Universidad de Chile. Chile.

4. Ayudante Alumno. Departamento de Patología Oral. Facultad de Odontología, Universidad de Chile. Chile.

5. Profesor Adjunto. Departamento de Patología Oral. Facultad de Odontología, Universidad de Chile. Chile.

6. Profesor Titular. Departamento de Cirugía Bucal y Máxilofacial. Facultad de Odontología, Universidad de Chile. Chile.
}

Correspondencia autor: Daniela Adorno Farias. daniadorno@gmail.com. Trabajo recibido el 02/11/2010. Aprobado para su publicación el 28/12/2010. 
Odontología de la Universidad de Chile, por presentar una lesión en lengua asintomática que ella reporta tener hace 8 meses. La paciente tiene hipertensión arterial controlada, sin hábitos relacionados con tabaco ni alcohol y relata antecedentes familiares de enfermedad cardiaca. Es desdentada total y portadora de prótesis total superior e inferior, las cuales se encuentran en buen estado, pero el ajuste de la prótesis inferior presenta interferencia por el contacto con la lesión en el lado derecho.

Clínicamente se observa lesión que compromete casi la totalidad del borde lateral derecho de la lengua, extendiéndose a cara ventral. En tercio medio y posterior la lesión es una placa blanquecina, homogénea, que no se desprende al raspado y con superficie lisa a rugosa. En el sector más anterior la lesión es exofítica, de base sésil, superficie rugosa, color de mucosa normal, con algunos sectores blanquecinos (Figura 1).

Se toma biopsia incisional en tres sectores de la lesión (Figura 2) y el examen histológico arrojó en el sector A la presencia de una proliferación epitelial bulbosa endofítica y exofítica, digitiforme, hiperortoqueratinizada con algunas células de citoplasma claro tipo coilocitos en el estrato espinoso. En el tejido conjuntivo se observó abundante infiltrado inflamatorio, predominantemente linfocitario. El diagnóstico fue de carcinoma verrucoso (Figura 3). En los sectores B y C se observó una leve alteración de la estratificación epitelial y fueron diagnosticados como displasia intraepitelial leve (Figura 4).

La paciente fue derivada al Hospital San Borja Arriarán donde se realizó la extirpación completa de la lesión y así se confirmó el diagnóstico inicial (Figura 5). Inicialmente, post intervención quirúrgica, la paciente presentó dislalia que fue mejorada con tratamiento de fonoaudiología. Después de tres años de realizada su cirugía, la paciente se encuentra en buen estado de salud, con buena movilidad lingual, sin dificultades para hablar o comer y sin recidiva de su enfermedad (Figura 6).

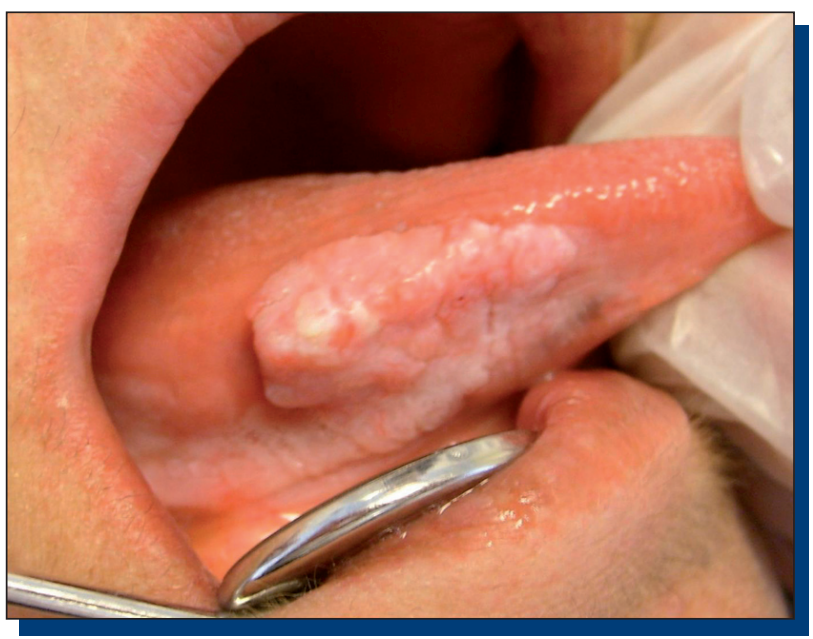

Figura 1. Lesión que compromete casi la totalidad del borde lateral derecho de lengua, extendiéndose a cara ventral.

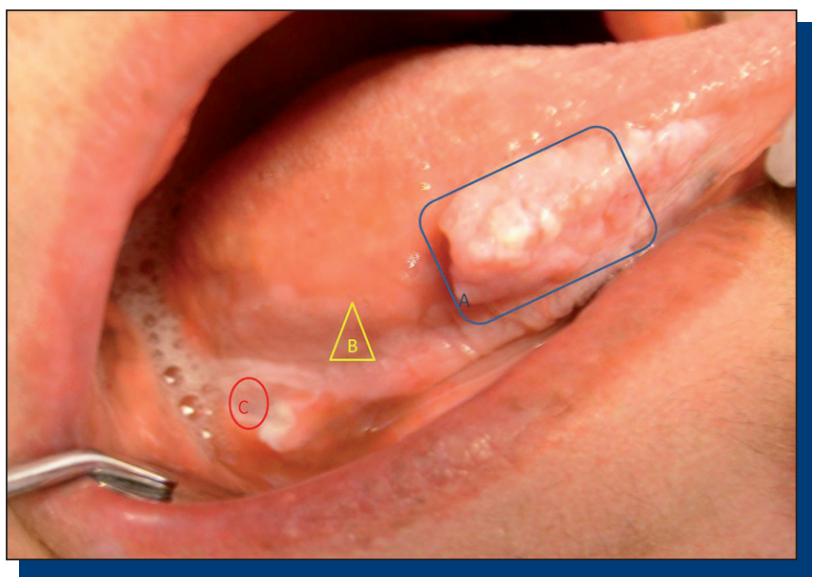

Figura 2. Sectores A, B y C corresponden a las zonas elegidas para la biopsia incisional.

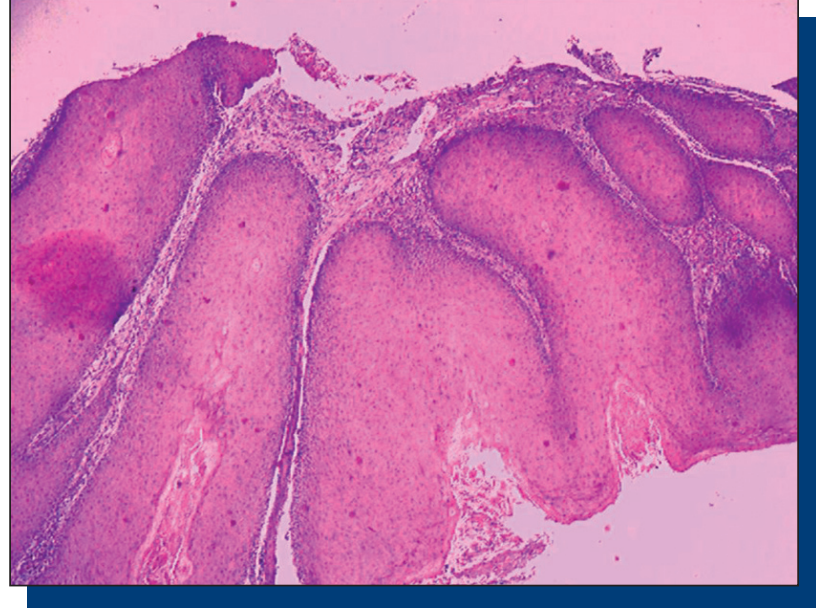

Figura 3. Zona de carcinoma verrucoso en la que se observa epitelio engrosado y papilas bulbosas. Tinción hematoxilina-eosina, aumento real 10X.

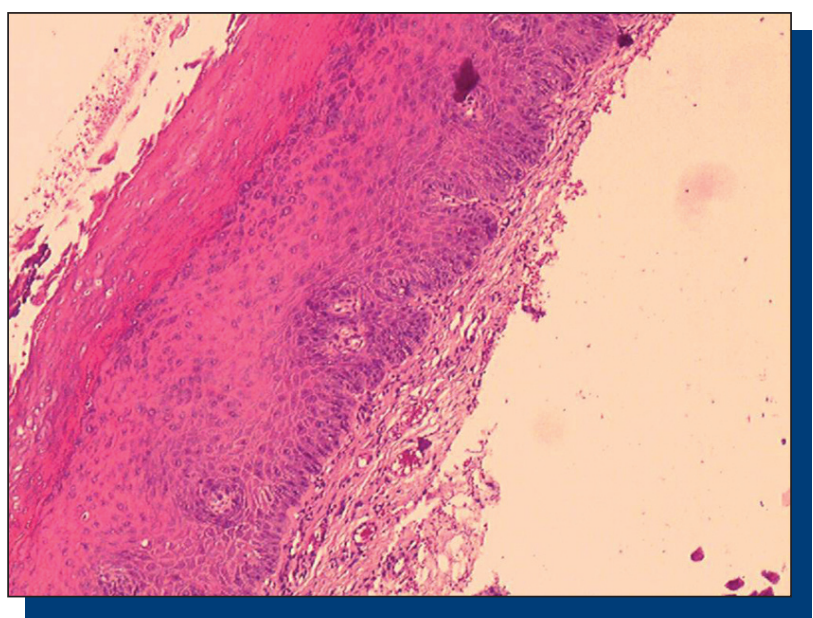

Figura 4. Zona de displasia leve con papilas bulbosas, alteración de la estratificación celular en estratos basal y parabasal. También se observa hiperortoqueratosis. Tinción hematoxilina-eosina, aumento original 40X.

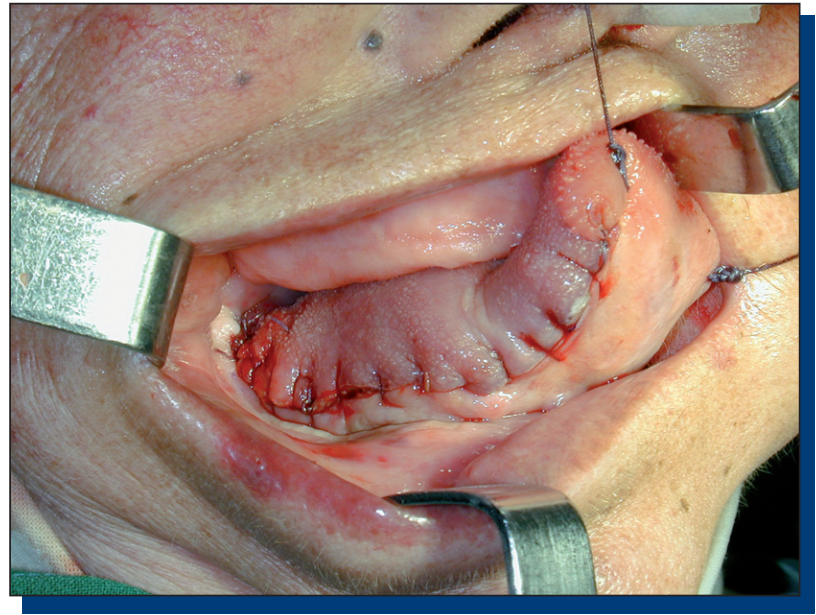

Figura 5. Imagen post excisión completa de la lesión. 


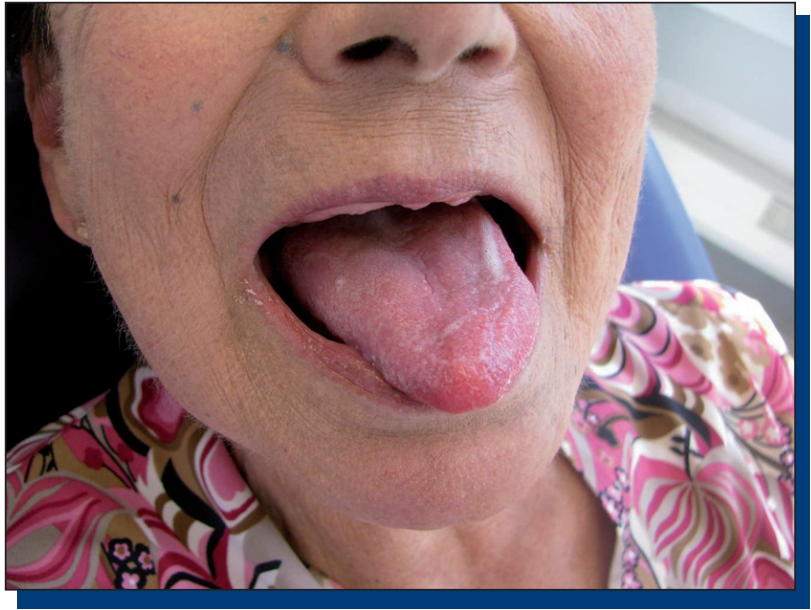

Figura 6. Paciente tras tres años de la cirugía, presentando funcionalidad normal de lengua.

\section{ASPECTOS DEMOGRÁFICOS E HISTOPATOLÓGICOS DE 20 CASOS DEL IREPO}

Al revisar la información en la base de datos del Instituto de Referencia en Patología Oral de la Universidad de Chile en el periodo comprendido entre enero de 1984 y octubre de 2010, se encontraron 20 casos de CV con un promedio de edad de 70.7 años (Tabla 1). El $60 \%$ de los casos fueron mujeres.

Las localizaciones preferentes en los casos revisados para este trabajo fueron (Tabla 1): encía inferior y lengua con un porcentaje similar de $27.3 \%$ de los casos, en segundo lugar encía superior y mucosa yugal, cada una con una frecuencia de $13.6 \%$ y por último labio inferior y paladar duro, con un porcentaje de $9.1 \%$ cada, como se resume en la Tabla 1 . De los 20 casos, solamente dos comprometen encía y mejilla simultáneamente.

Tabla 1. Resumen de datos clínicos de pacientes con carcinoma verrucoso revisados en la base de datos del IREPO en el periodo enero de 1984 a octubre de 2010.

\begin{tabular}{|c|c|c|c|}
\hline \hline $\mathbf{N}^{\circ}$ & Edad & Género & Localización \\
\hline 1 & 64 & $\mathrm{~F}$ & Encía inferior \\
\hline 2 & 71 & $\mathrm{~F}$ & Lengua \\
\hline 3 & 83 & $\mathrm{M}$ & Encía inferior \\
\hline 4 & 68 & $\mathrm{~F}$ & Encía inferior y mejilla \\
\hline 5 & 56 & $\mathrm{M}$ & Encía superior \\
\hline 6 & 73 & $\mathrm{~F}$ & Lengua \\
\hline 7 & 49 & $\mathrm{~F}$ & Labio inferior \\
\hline 8 & 79 & $\mathrm{M}$ & Encía inferior \\
\hline 9 & 66 & $\mathrm{M}$ & Lengua \\
\hline 10 & 86 & $\mathrm{~F}$ & Encía superior \\
\hline 11 & 75 & $\mathrm{~F}$ & Encía superior \\
\hline 12 & 79 & $\mathrm{~F}$ & Paladar duro \\
\hline 13 & 69 & $\mathrm{~F}$ & Encía inferior y mejilla \\
\hline 14 & 77 & $\mathrm{~F}$ & Mejilla derecha \\
\hline 15 & 65 & $\mathrm{M}$ & Labio inferior \\
\hline 16 & 80 & $\mathrm{M}$ & Paladar duro \\
\hline 17 & 77 & $\mathrm{M}$ & Lengua \\
\hline 18 & 69 & $\mathrm{~F}$ & Lengua \\
\hline 19 & 60 & $\mathrm{M}$ & Lengua \\
\hline 20 & 68 & $\mathrm{~F}$ & \\
\hline & & & \\
\hline
\end{tabular}

\section{DISCUSIÓN}

El carcinoma verrucoso fue descrito por Ackerman en $1948^{(4)}$ como un tipo de carcinoma de células escamosas con características clínicas e histológicas particulares. La prevalencia del CV entre los tipos de CCE ha sido reportada entre valores de 2 y $4.5 \%{ }^{(5)}$. Un estudio realizado en Brasil reportó una frecuencia entre 0.53 y $0.6 \%$ de $\mathrm{CV}$ en un total de 3.500 casos de CCE bien diferenciados ${ }^{(6)}$. La frecuencia de esta patología en India es la más alta al compararla con otros países, llegando a cifras como la reportada por Rekha et al. $(2010)^{(2)}$, de $16.08 \%$ en un total de 827 casos de $\mathrm{CCE}^{(2)}$.

Los 20 casos de CV encontrados presentaros un promedio de edad de 70.7 años, lo que coincide con lo reportado en la literatura. $\mathrm{E}$ mayor porcentaje de CV observado en mujeres difiere un poco de la literatura que reporta una predilección por el sexo masculino, reportando una diferencia entre hombres y mujeres de hasta 3.4:1, entre la sexta y séptima década.

Las localizaciones preferentes en los casos revisados para este trabajo fueron encía inferior y lengua, luego encía superior y mucosa yugal y por último labio inferior y paladar duro, las que coinciden con las reportadas en la literatura, sin embargo mucosa yugal tiende a ser el sitio más prevalente y lengua en general ocupa la tercera o cuarta posición en prevalencia ${ }^{(2,6,7)}$. El compromiso de dos zonas simultáneamente como fue encía y mejilla, ha sido reportado previamente dado el carácter proliferativo de esta lesión ${ }^{(2,7)}$.

La etiología del CV no está completamente establecida, pero se sugieren factores de riesgo como el uso del tabaco y actividad viral oportunista asociado al papiloma virus humano $(1,2,6,7,8,9)$. En un estudio realizado en India, país con más alta prevalencia de $\mathrm{CV}$, se observó que de un total de 133 casos con CV, el $69.9 \%$ de los pacientes presentaban hábito de mascar tabaco, con o sin otros factores de riesgo asociados como fumar y consumir alcohol(2). Se ha descrito que solamente de un 20 a $30 \%$ de los pacientes no presentan hábitos asociados, lo que implica que otros factores son responsables por el desarrollo de estos carcinomas ${ }^{(2)}$. En el presente caso la paciente no relata hábitos relacionados con el tabaco en ninguna etapa de su vida y el único factor local presente que se puede asociar es ser portadora de prótesis total. La prótesis inferior se encontraba con interferencia y consecuente desajuste por la presencia de la lesión.

La asociación clínica del carcinoma verrucoso y leucoplasia es significante y evidencias indican que una leucoplasia no tratada puede desarrollar un carcinoma verrucoso ${ }^{(8,10)}$. De esta forma se requiere una biopsia en lesiones blanquecinas de la mucosa, una vez que estas tienen una gran variedad de posibles diagnósticos, incluyendo una hiperqueratosis, displasia epitelial en distintos grados y carcinoma de células escamosas ${ }^{(8)}$. En el caso presentado se observó una lesión extensa en borde lateral y cara ventral de lengua con un aspecto clínico heterogéneo. Por este motivo se decidió realizar biopsia incisional de tres sectores de esta lesión y la histología confirmó que en los dos sectores en que el aspecto de la lesión era de una placa blanquecina de superficie lisa se observó una displasia epitelial. Por otro lado, el sector con aspecto exofítico y superficie rugosa correspondió a un carcinoma verrucoso.

Aunque el diagnóstico histológico de la biopsia incisional sea un carcinoma verrucoso, no se puede descartar que al extirpar la lesión completamente existan zonas adyacentes de carcinoma de células escamosas de la variedad convencional, cambiando el diagnóstico, tratamiento y pronóstico para el paciente. Ogawa et al..$^{(7)}$ reportan que de los 15 pacientes con el resultado de un carcinoma verrucoso en la primera biopsia, en 3 casos (20\%) se confirmó un diagnóstico de carcinoma de células escamosas en el examen de la pieza quirúrgica completa(7).

Con el ejemplo del caso reportado, se trata de dar énfasis a la importancia de una adecuada selección del sector a ser biopsiado y que en lesiones con características clínicas heterogéneas se hace necesario programar biopsia incisional en distintos sectores, por la variedad de diagnósticos que pueden estar asociados.

En el caso tratado se realizó la extirpación quirúrgica de la lesión sin asociación con radioterapia, tratamiento largamente defendido en la literatura que se relaciona con una tasa de $77.6 \%(3)$ a $88.9 \%{ }^{(7)}$ de supervivencia a los 5 años. Ogawa et al. ${ }^{(7)}$ sugieren que la mayoría de los casos pueden ser controlados solamente con intervención quirúrgica, una vez que encontró positividad de CD44v9, que es una molécula de adhesión celular variante 9, cuya expresión está relacionada con la disminución de diferenciación en la mayoría de los casos de $\mathrm{CV}^{(7)}$.

La tasa de recurrencia varía en la literatura desde un 6.12 al $40 \%$ y algunos autores defienden que las altas tasas estarían relacionadas a que el crecimiento lento y bajo potencial de invasión del CV puede conducir a un tratamiento quirúrgico inadecuado, con márgenes quirúrgicos comprometidos(6).

Tras tres años de tratamiento la paciente sigue en control, se encuentra en excelentes condiciones de salud y no presenta recidivas, ni metástasis. Actualmente la paciente no hace uso de prótesis inferior porque tras la extirpación de la lesión se perdió retención para la prótesis del sector posterior y se indicó la asociación de rehabilitación con implantes y la paciente no quiere realizar el tratamiento indicado. 
En conclusión, es importante destacar que por el excelente pronóstico de esta patología un correcto plan de tratamiento del paciente, con adecuada biopsia incisional y diagnóstico histológico apropiado, se puede prevenir que la lesión se extienda a otras áreas adyacentes, comprometiendo una menor área anatómica tras la intervención quirúrgica.

\section{REFERENCIAS BIBLIOGRÁFICAS}

1. World Health Organization. Classification of Tumours. Pathology \& Genetics. Head and neck Tumours, Lyon 2005: 122-123.

2. Rekha KP, Angadi PV. Verrucous carcinoma of the oral cavity: a clinico - pathologic appraisal of 133 cases in Indians. Oral Maxillofac Surg, 2010; 14(4): 211-218.

3. Walvekar RR, Chaukar DA, Deshpande MS, Prathamesh SP, Chaturvedi P, Kakade A, Kane SV, D'Cruz AK. Verrucous carcinoma of the oral cavity: a clinical and pathological study of 101 cases. Oral Oncology, 2009; 45: 47-51.

4. Ackerman LV. Verrucous carcinoma of the oral cavity. Surgery, 1948; 23: $670-678$.

5. Batsakis JG, Hybels R, Crissman JD, Rice DH. The pathology of head and neck tumors: verrucous carcinoma. Head Neck Surg, 1982; 5: 29-38.

6. Oliveira DT, De Moraes RV, Fiamengui JF, Neto JF, Landman G, Kowalski LP. Oral verrucous carcinoma: a restropective study in São Paulo region, Brazil. Clin Oral Invest, 2006; 10: 205-209.
7. Ogawa A, Fukuta Y, Nakajima T, Kanno SM, Obara A, Nakamura K, Mizuki H, Takeda Y, Satoh M. Treatment resoults of oral verrucous carcinoma and its biological behavior. Oral Oncology, 2004; 40: 793797.

8. Alkan A, Bulut E, Gunhan O, Ozden B. Oral verrucous carcinoma: a study of 12 cases. Eur J Dent, 2010; 4: 202-207.

9. Sorger K, Myrden JA. Verrucous carcinoma of the buccal mucosa in tobacco-chewers. Canad M A J, 1960; 83: 1413-1417.

10. Bagan J, Scully C, Jiménez Y, Martorell M. Proliferative verrucous leukoplakia: a concise update. Oral Diseases, 2010; 16: 328-332. 